\title{
BRECHA DE GÉNERO EN EL MERCADO LABORAL COLOMBIANO EN TIEMPOS DE LA COVID-19*
}

\author{
Recibido: 1 de septiembre de 2020 - Aprobado: 6 de noviembre de 2020 \\ https://doi.org/10.22395/seec.v23n55a13 \\ Cristina Isabel Ramos Barroso** \\ Maria Cristina Bolivar Restrepo ${ }^{* * *}$
}

\section{RESUMEN}

El propósito de este trabajo consiste en analizar el impacto que ha traído la crisis de salud pública mundial derivada de la COVID-19, para la mujer y su rol en el mercado laboral colombiano. El estudio busca evaluar la dinámica de los principales indicadores laborales en hombres y mujeres, y verificar si la emergencia sanitaria ha contribuido a ampliar la brecha de género en el mercado de trabajo. La estrategia empírica consiste en un análisis estadístico sobre los principales indicadores laborales extraídos de la Gran Encuesta Integrada de Hogares (GEIH) para los años 2019 y 2020, elaborada por el Departamento Administrativo Nacional de Estadísticas (DANE), así como la revisión documental sobre las principales vertientes existentes en los estudios de género y el mercado laboral. Como principal hallazgo se tiene que en la mayoría de los indicadores laborales, en particular la tasa de desempleo, inactividad y sectores críticos, la COVID-19 ha contribuido al incremento de la brecha laboral entre hombres y mujeres en el mercado de trabajo.

\section{PALABRAS CLAVE}

Brecha de género; mercado laboral; COVID-19; discriminación laboral; mujer; Colombia.

\section{CLASIFICACIÓN JEL}

$\mathrm{J} 71, \mathrm{~J} 40$

\section{CONTENIDO}

Introducción; 1. Antecedentes; 2. Marco teórico; 3. Algunos estudios sobre la brecha de género en el mercado laboral en tiempos de la COVID-19; 4. Metodología; 5. Resultados; 6. Conclusiones; Referencias.

Este artículo es producto del trabajo de investigación en la maestría de Ciencias Económicas, Universidad Nacional de Colombia, Medellín.

* Profesional en Negocios Internacionales, Universidad de Medellín, Medellín, Colombia. Estudiante de la Especialización en Docencia y Gestión de la Educación Superior, Universidad de Medellín, Medellín, Colombia. Maestrando en Ciencias Económicas, Universidad Nacional de Colombia, Medellín, Colombia. Asistente editorial revista Semestre Económico, Universidad de Medellín, Medellín, Colombia. Correo electrónico: cramosb@unal. edu.co; isabelbarroso92@gmail.com. Orcid: https://orcid.org/0000-0002-3912-1905

... Ingeniera Administradora, Universidad Nacional de Colombia, Medellín, Colombia. Maestrando en Ciencias Económicas, Universidad Nacional de Colombia, Medellín, Colombia. Analista de procesos, entrega y activación de tarjetas de crédito, Compañía de Financiamiento Tuya, Medellín, Colombia. Correo electrónico: mcbolivarr@ unal.edu.co; mcbolivarr@gmail.com. Orcid: https://orcid.org/0000-0002-1996-4219 


\title{
GENDER GAP AND THE COLOMBIAN LABOR MARKET IN THE TIME OF COVID-19
}

\begin{abstract}
The purpose of this work is to analyze the impact of the global public health crisis derived from COVID-19 on women and their role in the Colombian labor market. The study tries to evaluate the dynamics of the main labor indicators in men and women, and to verify whether the health emergency has contributed to widening the gender gap in the labor market. The empirical strategy consists of a statistical analysis on the main labor indicators from the Great Integrated Household Survey (GEIH) for the year 2020, by the National Administrative Department of Statistics (DANE), as well as the documentary review on the main aspects existing in gender studies and the labor market. The main finding is that in most labor indicators, in particular the unemployment rate, inactivity rate and critical sectors, COVID-19 has contributed to increase the labor gap between men and women in the labor market.
\end{abstract}

\section{KEYWORDS}

Gender Gap; Labor Market; COVID-19; Labor Discrimination; Woman; Colombia.

\section{JEL CLASSIFICATION}

$\mathrm{J} 71, \mathrm{~J} 40$

\section{CONTENT}

Introduction; 1. Background; 2 . Theoretical framework; 3 . Some studies on the gender gap in the labor market in the time of COVID-19; 4. Methodology; 5. Results; 6. Conclusions; References.

\section{A DESIGUALDADE DE GÊNERO E O MERCADO DE TRABALHO COLOMBIANO NA ÉPOCA DA COVID-19}

\section{RESUMO}

O objetivo deste trabalho é analisar o impacto que a crise global de saúde pública derivada da Covid-19 teve sobre as mulheres e seu papel no mercado de trabalho colombiano. O estudo busca avaliar a dinâmica dos principais indicadores laborais em homens e mulheres e verificar se a emergência sanitária tem contribuído para o aprofundamento da desigualdade de gênero no mercado de trabalho. A estratégia empírica consiste na análise estatística dos principais indicadores laborais extraídos da GEIH para o ano 2020, elaborado pelo DANE, e a revisão documental sobre os principais aspectos existentes nos estudos de gênero e no mercado de trabalho. A principal conclusão é que na maioria dos indicadores de trabalho, em particular a taxa de desemprego, inatividade e setores críticos, a Covid-19 tem contribuído para um aumento da desigualdade entre homens e mulheres no mercado de trabalho.

\section{PALAVRAS CHAVE}

Desigualdade de gênero; mercado do trabalho; Covid-19; discriminação; mulher; Colômbia.

\section{CLASSIFICAÇÃO JEL}

$\mathrm{J} 71, \mathrm{~J} 40$

\section{CONTEÚDO}

Introdução; 1. Antecedentes; 2. Referencial teórico; 3 . Alguns estudos sobre a disparidade de gênero no mercado de trabalho na época da Covid-19; 4. Metodologia; 5. Resultados; 6. Conclusões; Referências. 


\section{INTRODUCCIÓN}

A pesar del incremento sustancial de la participación de las mujeres en el mercado laboral en las últimas décadas, la población femenina continúa experimentando problemas de desigualdad, inequidad e invisibilidad; fruto de la construcción histórico cultural que ha asignado roles, atributos y responsabilidades a la mujer de acuerdo con su condición biológica y reproductiva.

A finales del 2019, las mujeres contaban con una tasa global de participación (TGP) de 53,1\%; posicionándose 20,7 puntos porcentajes (pps) menor a los de su contraparte masculina. Cuando las mujeres logran ingresar al mercado laboral, experimentan una tasa de desempleo más alta que los hombres, llegando a una brecha de 5,35 pps entre ambos. Se debe destacar que durante los últimos diez (10) años, la brecha entre la tasa de desempleo femenina y masculina solo ha disminuido en 1,05 pps; a favor de los hombres (DANE, 2019). Por su parte, las mujeres ocupadas se encuentran mayoritariamente en sectores económicos que tradicionalmente han sido inestables, desprotegidos y en condiciones laborales precarias tales como: el trabajo doméstico, comercio, atención a salud humana, educación, etc. Solo para el empleo doméstico, las diferencias son abismales: el 96,5\% de los trabajadores domésticos son mujeres. Adicional, la tasa de informalidad femenina llegó a 48,7 \%, mientras que los hombres reportaban un $44,2 \%$ durante el mismo periodo. Las actividades económicas en donde se concentra la mayoría de la población femenina, descritas anteriormente, se caracterizan por ser al mismo tiempo informales (DANE, 2019)'.

De acuerdo con el DANE y ONU (2019), las brechas entre salarios oscilan entre 37 pps y 19 pps de acuerdo con el nivel educativo para el año 2019. Si tanto el hombre como la mujer tienen menor nivel educativo, esta última recibiría sólo 63 pesos por cada 100 pesos que recibe su contraparte. En caso contrario, si ambos cuentan con un nivel alto de educación, las mujeres reciben 81 pesos por cada 100 pesos que recibe el hombre.

Dentro de un contexto económico de crecimiento, las condiciones del mercado laboral favorecen a la población masculina. En el presente estudio se analizará el impacto que ha traído la crisis de salud pública mundial derivada de la COVID-19, para la mujer y su rol en el mercado laboral colombiano. La metodología implementada consiste en un análisis estadístico sobre los principales indicadores laborales extraídos de la Gran Encuesta Integrada de Hogares (GEIH) para el año 2020, elaborada por el Departamento Administrativo Nacional de Estadísticas (DANE), así como la revisión documental sobre las principales vertientes en los estudios de género.

Los indicadores del mercado laboral fueron extraídos del Departamento Administrativo Nacional de Estadística, los cuales tienen definidos unas metodologías especificas para cada indicador. 
Como principal hallazgo se tiene que, en la mayoría de los indicadores laborales, en particular la tasa de desempleo, inactividad y sectores críticos, la COVID-19 ha incrementado la brecha laboral entre hombres y mujeres en el mercado de trabajo.

El presente trabajo consta de cinco secciones, en la primera sección se presenta la descripción de la problemática que se quiere analizar; en la segunda, el marco teórico, se realiza una revisión de los estudios a nivel nacional sobre las brechas de género dentro del mercado laboral; en la tercera sección se encuentran algunas investigaciones sobre estas brechas de género, pero en tiempos de la COVID-19; en la cuarta se especifica la estrategia empírica con la que se analizará el impacto de la pandemia mundial de salud en las brechas de género; en la quinta sección se presentan los resultados, con el fin de identificar los cambios que ha tenido las brechas de género durante la pandemia de la COVID-19; y finalmente, en la sexta sección se muestran las principales conclusiones del trabajo.

\section{ANTECEDENTES}

Dimensionar los efectos de la COVID-19 en la economía global y lidiar con su impacto en el aparato productivo agregado, se ha convertido en un imperativo dentro de las agendas gubernamentales de los países en los últimos meses. Los eventos que trajo consigo la pandemia mundial afectaron significativamente las perspectivas de crecimiento de la economía. En términos globales, de acuerdo con las proyecciones del Fondo Monetario Internacional (IMF), la economía global se contraerá en un aproximado de $3 \%$ en 2020, mientras que las proyecciones para Colombia apuntan a una contracción del 2,4 \% del producto nacional (IMF, 2020). Si bien la economía colombiana muestra signos de resiliencia frente a la crisis, otro panorama distinto y muy relevante como lo es el mercado laboral, revela señales de estancamiento, en particular el mercado de trabajo femenino.

Diversos estudios han analizado las características y los indicadores de empleo femenino, encontrando que tradicionalmente, este grupo poblacional, presenta cifras de empleo más bajas en comparación con su contraparte masculina, y es quien sufre con mayor rigor los efectos de las crisis económicas (Flores y Salas, 2015; Torns y Recio, 2012; Cerquera-Losada et al., 2019; Cerquera-Losada at al., 2020). Bajo esta premisa, y dada la naturaleza de esta crisis, donde se han visto afectados sectores intensivos en mano de obra femenina como la salud, es posible que los efectos de la COVID-19 contribuyan a ampliar la brecha de género ya existente.

El análisis de género trasciende el mercado de trabajo, las diferencias de género radican, si se quiere, en un enfoque social, al ser las mujeres las que por tradición se 
ocupan de las tareas del hogar, el cuidado de los niños y otros trabajos no remunerados. Este es un tema importante a resaltar, y es el hecho de que los trabajos no remunerados en las mujeres como los cuidados en el hogar se han incrementado, como consecuencia de la cuarentena implementada en muchos países (Farré et al., 2020; Alon et al., 2020). Las mujeres suelen ser las principales cuidadoras en el hogar y, en general, tienen a cargo más tareas domésticas y responsabilidades en relación con los hombres. Según los datos del UNDP (2020) las mujeres pasan en promedio 2,5 veces más tiempo en el trabajo de cuidado no remunerado. Ahora bien, con las escuelas cerradas, el creciente número de personas contagiadas en muchos países y la necesidad de cuidar a pacientes y niños en el hogar, la carga de trabajo no remunerado para las mujeres probablemente aumente, lo que tendrá un fuerte impacto en la participación de la mujer en el mercado laboral en corto plazo.

Bajo este panorama, profundizar en las consecuencias que ha traído consigo la crisis de salud pública mundial derivada de la COVID-19 no sólo es fundamental para las economías actuales, sino que se convierte en una obligación social a favor de la igualdad y equidad de género.

\section{MARCO TEÓRICO}

En Colombia, los estudios sobre las brechas de género han demostrado que las mujeres siguen obteniendo resultados más desventajosos que los hombres en el mercado laboral. Las fuentes de dicha desigualdad varían según el análisis, pero los factores más determinantes apuntan a una discriminación de género que nace de una construcción histórica que asigna roles, atributos y responsabilidades a la mujer por su condición biológica. En la presenta sección se abordarán los tres tipos de discriminación contra la mujer más estudiados en la literatura de género, los cuales han persistido a través del tiempo y son un obstáculo para el empoderamiento económico, social y profesional de la mujer.

\subsection{Sobre la discriminación salarial}

Una de las principales evidencias sobre la existencia de discriminación laboral de género se encuentra sustentada en la persistente brecha salarial o de remuneración entre hombres y mujeres. En Colombia, los estudios sobre las diferencias salariales han intentado explicar, desde diferentes metodologías, los factores que más influyen en este fenómeno, tales como: las diferencias en capital humano, la segregación horizontal y vertical entre ambos géneros, la división sexual del trabajo, entre otras causas. 
Una de las investigaciones realizadas en el país con este propósito fue adelantada por Galvis (2010), en la cual se profundiza en las brechas salariales de acuerdo al género en las diferentes ciudades del país. A través de la descomposición de Blinder-Oaxaca en el contexto de regresión por cuantíles, el autor concluye que las diferencias salariales, a favor de los hombres, no solo se encuentran explicadas por los atributos observables de los individuos, sino también por otros elementos tales como las habilidades innatas, la motivación o decisión de participar en el mercado de trabajo. Adicional, se encuentra que, las brechas salariales se amplían en el grupo de personas con ingresos más bajos, especialmente en las ciudades más apartadas de la actividad económica del país. Por tanto, la población femenina tendría una desventaja adicional sobre su salario si labora en dichos lugares.

Un análisis más reciente realizado por Cerquera-Losada et al. (2020), analiza las causas de las brechas de género en el país y en el departamento de Caldas, desde la teoría del capital humano. Utilizando la descomposición de Blinder y Oaxaca, los autores encuentran que hay una diferencia salarial de $27,31 \%$ a favor del hombre, incluso cuando las mujeres presentan mayores características productivas como el en nivel de educación y experiencia. Finalmente, aunque no se puede concluir con toda certeza que el total del diferencial salarial entre hombres y mujeres sea producto de la discriminación en contra de las mujeres; los autores consideran que existen algunas características naturales de la mujer, como la maternidad, que generalmente son vistas como un gasto para los empleadores, que pueden traducirse en salarios más bajos.

Mejía-Lleras (2018), por su parte, realizó un análisis comparativo de brechas salariales a través de diferentes metodologías estadísticas propuestas por Mincer (1974), Koenker y Basset (1978), y Oaxaca y Blinder (1973). En todas las metodologías presentadas, los resultados indican que existen diferencias de salario a favor de la población masculina, y que esta diferencia se amplía en los grupos de personas en lo más bajo y lo más alto de la distribución de ingresos, confirmando la existencia de un efecto de "pisos pegajosos" y "techos de cristal"

\subsection{Sobre la discriminación ocupacional}

Otro escenario común de discriminación por género se sustenta desde la segregación horizontal y vertical dentro de las estructuras organizacionales. Resultados empíricos

2 Pisos pegajosos es un concepto que se utiliza para hacer referencia a las mujeres que tienen un bajo grado de empoderamiento económico, que enfrentan obstáculos estructurales en el acceso al empleo o cuya inserción laboral se da en trabajos precarios. Techos de cristal, se refiere a las mujeres con altos niveles de empoderamiento económico y de educación e inserción laboral, pero que enfrenta barreras invisibles que le impiden ascender hacia los niveles superiores de la escalera corporativa. 
han demostrado que las mujeres permanecen en la base de las organizaciones, en cargos de menor movilidad y ritmos de ascensos más lentos. Para las mujeres en escenarios de techos de cristal la desventaja parece ser mayor, las barreras invisibles que enfrentan para alcanzar cargos directivos limitan su desarrollo profesional y empoderamiento económico.

En un análisis realizado por Meza-Martínez (2018), se encuentra que, desde la segregación horizontal, aún persisten las barreras socio-culturales que mantienen feminizados algunos cargos a partir de estereotipos de género, como el caso de los servicios generales, secretarias, asistentes y enfermerías; que como se ha mencionado en repetidas ocasiones, se valoran menos en las estructuras organizacionales. Para las mujeres en escenarios de techos de cristal se siguen manteniendo dificultades para el acceso de cargos de alta responsabilidad (segregación vertical), producto del imaginario de que los hombres tienen mayores competencias para ejercer autoridad.

Otro estudio realizado por Avendaño-Gelves (2011), para los años 2001, 2005 y 2009, demuestra que, durante los tres años analizados, se evidencia una segregación horizontal en la mayoría de las actividades económicas, siendo mayores las diferencias en la construcción, agricultura y transporte; que culturalmente han sido labores exclusivas para el género masculino. Uno de los elementos a destacar en la investigación es que, la segregación horizontal sí genera brechas salariales, pues las ocupaciones en las que la mujer se encuentra sobrerrepresentada, han sido históricamente, las más precarias, con peores condiciones laborales, pocas posibilidades de desarrollo y crecimiento y, sobre todo, de menor reconocimiento.

\subsection{División sexual del trabajo}

Tanto la discriminación salarial como la discriminación ocupacional han sido analizados, en parte, desde la división sexual del trabajo, que como se ha mencionado anteriormente, asigna roles, responsabilidades y tareas a la mujer por su condición de género (Lamas, 1996, citado en Meza-Martínez, 2018). Esta asignación se convierte en el criterio básico que se utiliza tanto en las organizaciones como en las familias, para el "reparto" desigual de funciones, donde los hombres son los agentes dominantes y ganadores de esta distribución.

Sobre el cuidado del hogar se puede destacar el estudio de Moreno (2017), quien examina la división social y sexual del trabajo doméstico y de cuidado no remunerado en la ciudad de Bogotá. Desde el análisis de la economía feminista, Moreno (2017) destaca la necesidad de reconocer las actividades domésticas y del cuidado como actividades impredecibles para el sistema económico actual, pues a 
pesar de aportar el $20 \%$ del Producto Interno Bruto, no son remuneradas y, sobre todo, aquellos quienes las llevan a cabo se encuentran catalogadas como "inactivas laboralmente". Uno de los resultados de la investigación muestra que la división sexual del trabajo se ha reconfigurado: tanto los hombres como mujeres asumen trabajos remunerados y no remunerados. A pesar de lo anterior, las mujeres cuentan con grandes limitaciones, condiciones laborales desventajosas y una carga mayor y más denigrante dentro de las tareas del hogar.

De igual manera, se resalta el trabajo de Mendoza y Melo (2019), cuyo propósito es analizar el peso de la maternidad en la determinación salarial y la relación entre maternidad e inserción laboral femenina en ocupaciones precarias. Los resultados del estudio demostraron el efecto positivo en la relación entre maternidad y las ocupaciones precarias, lo que significa que, a mayor número de hijos, mayor es la probabilidad de ejercer en condiciones precarias, con salarios menores. Solamente ser madre en Colombia significaría un incremento de $21 \%$ en la probabilidad de pertenecer a trabajos en dichas condiciones. Además, el incremento de un hijo puede aumentar la probabilidad en un $12 \%$ más.

\section{ALGUNOS ESTUDIOS SOBRE LA BRECHA DE GÉNERO EN EL MERCADO LABORAL EN TIEMPOS DE LA COVID-19}

Algunos trabajos en el contexto internacional han analizado el impacto de la crisis sanitaria derivada de la COVID-19 en el mercado laboral femenino; no obstante, para el caso de Colombia los datos son limitados por lo que se abordarán algunos ejercicios de corte internacional.

En un estudio elaborado por Alon et al., (2020) se analiza cómo la actual recesión económica causada por la COVID-19 afecta la desigualdad de género en el mercado laboral, tanto a corto como a largo plazo. Los autores usan datos sobre la distribución de mujeres y hombres en diferentes ocupaciones en Estados Unidos, y datos sobre el uso del tiempo y la división del trabajo en las tareas domésticas. Como principal conclusión los autores encuentran que, a corto plazo, la pandemia tendrá un efecto negativo en las oportunidades de empleo para las mujeres en comparación con los hombres. Este hallazgo se basa en dos premisas dispuestas por los autores: por un lado, la crisis actual es diferente a otras (tiene un origen diferente a otras crisis) y tiene un gran impacto negativo en las ocupaciones de servicios caracterizadas por altas cuotas de mano de obra femenina, como restaurantes y hoteles; y por otro lado, en el transcurso de la pandemia, la mayoría de los países han decidido cerrar las escuelas y las guarderías, lo que ha derivado en un aumento dramático del cuidado infantil y trabajo doméstico no remunerado. 
En un estudio de la Organización para la Cooperación y el Desarrollo Económicos (OECD) para la región MENA ${ }^{3}$ se analiza el impacto de la COVID-19 en la igualdad de género. Aunque el trabajo se enfoca en varias dimensiones, se resaltan los efectos de la pandemia en la economía y el mercado de trabajo femenino. Entre las principales conclusiones se encontró que si bien las consecuencias económicas de la crisis sanitaria se verán en todos los trabajadores y empresas por igual, la evidencia de crisis anteriores provocadas por enfermedades infecciosas apunta a efectos más agudos en las mujeres. Otro de los hallazgos tiene que ver con la mayor vulnerabilidad de las mujeres jóvenes en el mercado laboral y la informalidad. Los autores sostienen que las mujeres en la economía informal son las más afectadas por la recesión económica. Aproximadamente 62 \% en la región son empleadas informales y suelen trabajar en sectores y empleos peor remunerados que su contraparte masculina; por tanto, la interrupción de la actividad resultante de la crisis afectará de manera desproporcionada los ingresos y la posición laboral de las mujeres en la región (OECD, 2020).

Mediante una encuesta en línea, Oreffice y Quintana-Domeque (2020) recopilan datos relacionados con el bienestar mental, las percepciones, el empleo, la salud y el comportamiento de 1.500 personas en el Reino Unido. El objetivo es evaluar las diferencias de género en los aspectos mencionados. Al realizar estimaciones con Minimos Cuadrados Ordinarios, los autores encuentran que, en el aspecto laboral, las mujeres tienen más probabilidades de perder su trabajo que los hombres y, a su vez, tienen mayor probabilidad de tener trabajos con más riesgo de contagio de coronavirus que los hombres. Como conclusión, el estudio sugiere sustanciales diferencias de género en lo relacionado con la vulnerabilidad a la pandemia, en particular en aspecto laboral y mental.

Farré et al., (2020) analizan el impacto de la crisis derivada de la COVID-19 en la desigualdad de género y el trabajo remunerado y no remunerado en España. Los resultados diferenciados por género, evidenciaron una mayor probabilidad en las mujeres de perder el empleo. La cuarentena establecida provocó un gran aumento en el cuidado de los niños y las tareas domésticas, y se encontró que los hombres aumentaron levemente su participación en las tareas del hogar y el cuidado de los niños, pero la mayor parte de la carga en estas labores recayó sobre las mujeres, que ya realizaban la mayor parte del trabajo doméstico antes de la crisis. En general, los

3 El término MENA es un acrónimo del inglés "Middle East and North Africa", que se refiera a la región del Medio oriente y norte de África. Esta región está compuesta por Argelia, Bahrein, Yibuti, Egipto, Irak, Jordania, Kuwait, Líbano, Libia, Mauritania, Marruecos, Omán, Autoridad Palestina, Qatar, Arabia Saudita, Siria, Túnez, Emiratos Árabes Unidos y Yemen. 
autores afirman que la crisis de la COVID-19 parece haber aumentado las desigualdades de género en el trabajo remunerado y no remunerado a corto plazo en el país.

Kristal y Yaish (2020) realizan un estudio con el objeto de analizar cómo la crisis sanitaria ha impactado el empleo y los ingresos de hombres y mujeres en Israel. Mediante una encuesta con un total de 2.040 respuestas y empleando analisis descriptivo, los autores concluyen que la proporción de mujeres que han perdido los empleos, producto de la pandemia, es mayor en comparación con los hombres, lo cual contribuye a incrementar la brecha salarial y la desigualdad.

Adams-Prassl et al. (2020) en un ejercicio para Estados Unidos, Reino Unido y Alemania, examinan el impacto de la COVID-19 en el mercado laboral con el objeto de identificar los grupos poblacionales que han sido golpeados por la emergencia sanitaria. Los resultados son bastante heterogéneos entre los países; en los Estados Unidos y Reino Unido, las mujeres y los trabajadores sin un título universitario mostraron mayor probabilidad de perder sus empleos, mientras que los grupos poblacionales más jóvenes evicenciaron mayor probabilidad de experimentar una caída en sus ingresos. Finalmente, un patrón común en los tres países analizados mostró que las mujeres y los trabajadores menos calificados son los más afectados por la crisis.

Se identifican, entonces, dos grandes vertientes en la literatura sobre el impacto de la COVID-19 en la brecha de género en el mercado laboral: incremento del trabajo doméstico y la mayor pérdida de puestos de trabajo por parte de las mujeres; no obstante, los hallazgos en el estudio de Hupkau y Petrongolo (2020) para Reino Unido, esbozan la probabilidad de una transformación en los roles de los padres, en los asociados al cuidado doméstico. De acuerdo con los autores, es posible que estos roles en el cuidado de los niños y las tareas domésticas se inviertan en los hogares donde la madre trabaja en un sector crítico (sector económico con excepciones e indispensable como la salud) y el padre se ve obligado a quedarse en casa debido a las medidas de distanciamiento social. Esta transformación podría liderar un cambio en las normas de género hacia roles más equitativos. En cuanto al empleo, los autores encuentran que las mujeres son más suceptibles a perder sus puestos de trabajo, debido a que gran parte de los sectores con frecuentes interacciones sociales, como los servicios, donde la mano de obra femenina es representativa, han sido los más afectados por la crisis.

El análisis realizado en este estudio, enfatiza en el impacto de la COVID-19 en la brecha de género en Colombia, análisis que no ha sido abordado de manera formal, salvo por estadísticas e informes que comunmente se encuentran. Por otro lado, 
con este estudio se busca actualizar el contexto de la mujer en tiempos de crisis como la que se vive en la actualidad y revisar si esta ha sido mayormente afectada.

\section{METODOLOGÍA}

Con el propósito de conocer la forma como la COVID-19 ha afectado el mercado laboral femenino y verificar si la emergencia sanitaria ha incrementado las brechas de género en Colombia, se utilizan datos agregados y microdatos de la Gran Encuesta Integrada de Hogares de 2019 y 2020, para los trimestres móviles de enero-marzo, febrero-abril, marzo-mayo y abril-junio, y se analiza la evolución de los distintos indicadores laborales entre hombres y mujeres ${ }^{4}$. A través de un análisis gráfico se abordarán cada uno de los indicadores laborales más relevantes de la GEIH según el género, esto es:

- Tasa de desempleo y brecha entre hombres y mujeres.

- Tasa de ocupación y brecha entre hombres y mujeres.

- Inactivos.

- Ramas de actividad económica.

El análisis de estos indicadores permitirá constatar si la crisis actual ha constribuido a elevar la desigualdad de género en el mercado laboral colombiano.

\section{RESULTADOS}

Las brechas en términos laborales para hombres y mujeres en Colombia han sido por mucho tiempo una de las principales características del mercado laboral. Las desigualdades han sido comprobadas por diversos estudios que analizan las grandes vertientes sobre discriminación en el mercado de trabajo: discriminación salarial, discriminación ocupacional y división sexual del trabajo (Garzón y Ulloa, 2016; Palacios, 2019; Bello y Sepúlveda, 2016; Martín, 2007; Fleta-Asín y Pan, 2017; Chacón-Bejarano y Vanegas-Triana, 2019; Cerquera-Losada et al., 2020).

La presente sección muestra los principales resultados extraídos de la GEIH para los años 2019-2020, donde se analiza la evolución de los principales indicadores laborales en hombres y mujeres, con el fin de evidenciar las brechas de género en tiempos de la COVID-19.

4 Se hacen uso de trimestres móviles puesto que las estadísticas oficiales del mercado laboral por género se muestran de esta forma, no hay disponibilidad de datos mensuales u otros. 


\subsection{Género y tasa de desempleo}

Colombia es uno de los países latinoaméricanos que presenta las mayores tasas de desempleo en la población femenina, y mayores brechas en las tasas de desempleo entre hombres y mujeres (Herrera-Idárraga y Tribín, 2020). La crisis económica derivada de la emergencia sanitaria por la COVID-19, ha golpeado fuertemente los mercados laborales de la región y el mundo, y Colombia no es la excepción, en particular si se tienen en cuenta las grandes disparidades en las condiciones y características laborales de hombres y mujeres, que ya existían antes de la crisis sanitaria actual.

La tasa de desempleo es uno de los principales indicadores laborales, pues permite conocer el nivel de desocupación del país, y emite señales sobre el estado de la actividad económica. Para el trimestre móvil enero-marzo de 2020, fecha en la cual la economía colombiana apenas comenzaba a recibir los primeros efectos de la pandemia global, la tasa de desempleo para las mujeres era de $16,36 \%$, mientras que la de los hombres rondaba los 9,83\%, es decir, la brecha de desempleo alcanzó los $-6,53 \%$. Al comparar cada uno de los trimestres móviles analizados con los mismos periodos de 2019, se puede ver en la Ilustración 1 como la brecha de desempleo ha aumentado drasticamente. Para el trimestre abril-junio de 2019, la brecha de desempleo se ubicó en $-4,95 \%$, mientras que para el mismo periodo de 2020 este indicador aumentó al ubicarse en $-7,25 \%$, para una diferencia de 2,3 puntos porcentuales.

Bajo este panorama, es posible que las medidas de confinamiento y la suspensión de muchas actividades económicas hayan provocado un aumento en el desempleo y la inactividad de las mujeres (sobre todo en aquellas que se encuentran en sectores vulnerables y no aptos para teletrabajo), debido en parte al incremento del cuidado doméstico y el trabajo no remunerado, que han sido características transversales a la pandemia global (Hupkau y Petrongolo, 2020).

Es importante destacar que, aunque la crisis económica generada por la COVID-19 ha tenido un impacto significativo tanto en hombres y mujeres, son estas últimas quienes sufren en mayor grado los efectos causados por la pandemia. En la Figura 2 se puede observar que para el mes de mayo del 2020, el 50,1\% de las personas que admiten haber perdido su empleo a causa de la pandemia son mujeres, mientras que los hombres ocupan el 49,9\%, generando una brecha de tan solo 0,3 pps. Sin embargo, el panorama parece tener un cambio bastante significativo sólo un mes después: el porcentaje de mujeres que pierde su empleo incrementa a $52,3 \%$, en comparación a su contraparte que disminuye a $47,7 \%$. La brecha de género para el mes d e junio incrementaría hasta un 4,5 pps. Lo anterior puede ser 
consecuencia de dos escenarios igual de desventajosos para la mujer: el empleo masculino se recupera de manera más rápida que el femenino, o el impacto de la COVID-19 a mediano plazo tiene impactos más profundos para la mujer.

Figura 1. Tabla de desempleo y brecha (pps)
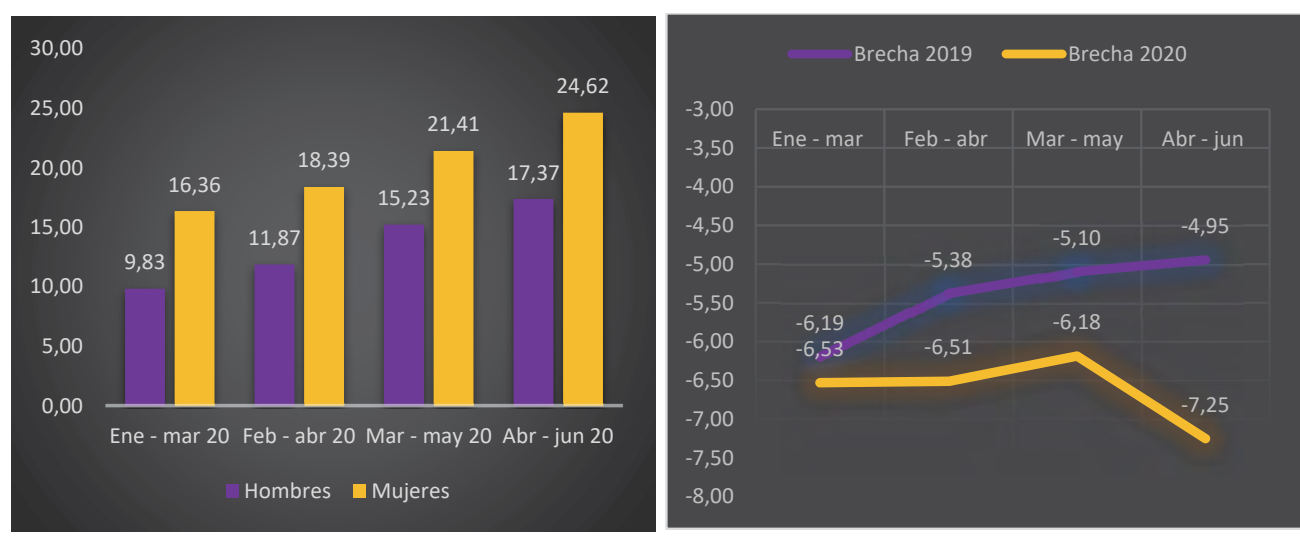

Fuente: elaboración propia con datos de la GEIH, DANE (2020).

Figura 2. Pérdida de empleo y/o fuente de ingresos a causa de la COVID-19

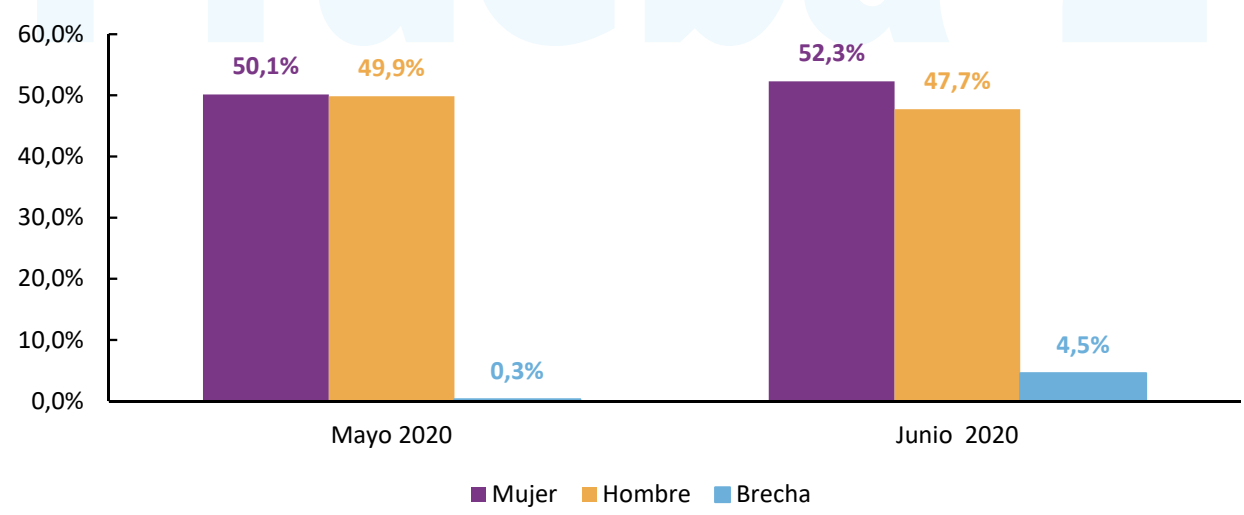

Fuente: elaboración propia con los microdatos de la GEIH, DANE (2020).

Nota: durante los meses de mayo y junio se adicionaron algunas preguntas en la GEIH, con el proposito de entender las dificultades que se han presentado debido a la pandemia mundial de la COVID-19. 


\subsection{Género, ocupación y salarios}

Frente a la tasa de ocupación, la Figura 3 muestra la evolución de este indicador para hombres y mujeres, así como la brecha. En primer lugar los datos evidencian un descenso agregado del nivel de ocupación en ambos sexos durante el período de análisis, no obstante ,en la mujer los efectos de esta reducción son mayores pues participan menos en el mercado laboral. En el caso de las mujeres, la tasa de ocupación se redujo en todos los periodos con respecto a 2019, la mayor reducción se dio en el trimestre abril-junio cuando pasó de 46,04 \% en 2019 a 33,1 \% en 2020. La brecha de ocupación entre 2019 y 2020 ha registrado leves disminuciones, sin embargo, la mujer sigue participando menos en el mercado laboral.

Figura 3. Tasa de ocupación y brecha (pps)
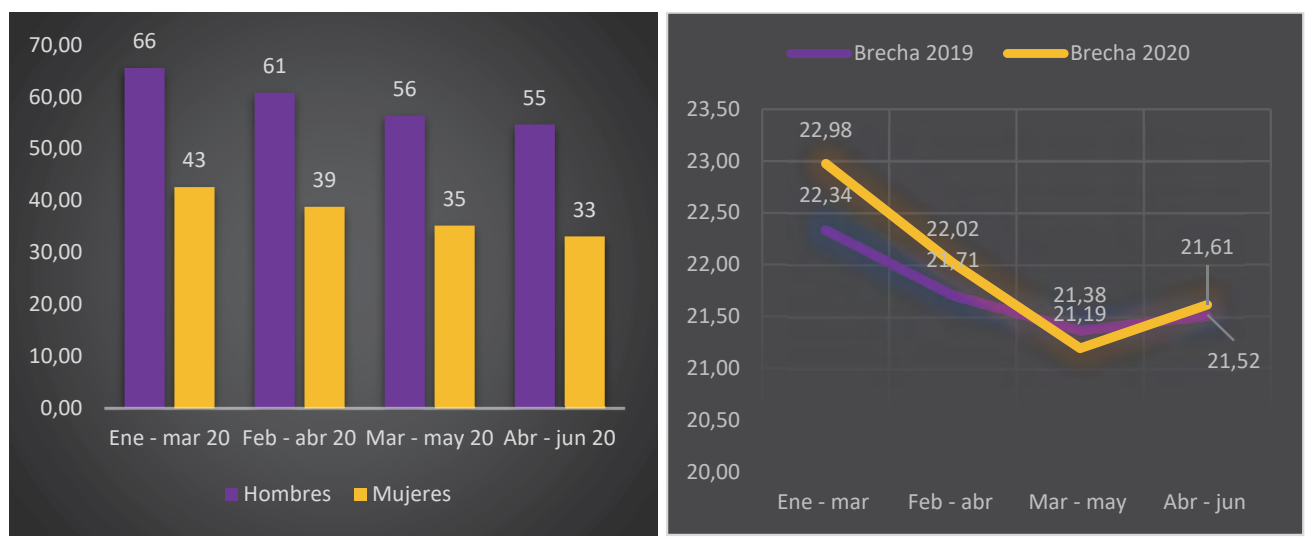

Fuente: elaboración propia con datos de la GEIH, DANE (2020).

Además de la ocupación, otro aspecto a rasaltar son los salarios. Como se puede apreciar en la Tabla 1, el promedio de la remuneración por hora para los trabajadores asalariados es ligeramente mayor para la población femenina en los tres meses analizados. Para enero y febrero, se observa que las mujeres ganan en promedio 2,8 \% y 4,6\% más que su contraparte. En junio, tiempos de la COVID-19, se logra percibir una disminución en el salario promedio de la población femenina, logrando una diferencia solo de 0,15\% entre ambos géneros (junio).

Por el contrario, para el primer cuartil de la distribución se logra observar unas diferencias significativas entre ambos géneros, obteniendo la población masculina mayor remuneración por hora. Para enero, los hombres ganaban en promedio 7,6 \% más que las mujeres, diferencia que disminuyó a 4,7 \% para el mes de febrero. Para el mes de Junio, durante la pandemia, la diferencia se volvió a ampliar llegando a 
un 10,5\%; siendo los hombres los beneficiados de dicha diferencia. Estos resultados comprueban que las brechas salariales se amplian en los grupos de menores ingresos, siendo las mujeres las más afectadas (Mejía, 2018; Galvis, 2010).

Para los grupos de mayores ingresos, no hay una tendencia muy clara. Para el primer mes analizado se encuentra que los hombres obtienen un 70 \% más que las mujeres por cada hora trabajada. Sin embargo, esta diferencia cambia para el mes de febrero, donde las mujeres obtienen 7 \% más remuneración que los hombres. Para junio, las cifras vuelven a invertirse, siendo la mujer quien gana sólo el $56 \%$ del ingreso por hora que gana su contraparte. Aún cuando las cifras son incomparables y pueden ser resultado de datos atípicos dentro de la muestra, las diferencias de ingreso por hora suelen ser más amplias cuando son los hombres quienes obtienen mayores ingresos.

Tabla 1. Remuneración por horas de asalariados antes y durante la COVID-19

\begin{tabular}{cccccccc}
\hline Mes & Género & Min & 1st Qu. & Median & Mean & 3rd Qu. & Max. \\
\hline \multirow{2}{*}{ Enero 2020 } & Mujer & 0 & 3,697 & 4,572 & 7,261 & 7,500 & 166,667 \\
& Hombre & 0 & 4,000 & 4,600 & 7,059 & 6,771 & 562,500 \\
\hline \multirow{2}{*}{ Febrero 2020 } & Mujer & 0 & 3,828 & 4,688 & 7,365 & 7,315 & 416,667 \\
& Hombre & 0 & 4,018 & 4,630 & 7,026 & 6,667 & 387,500 \\
\hline \multirow{2}{*}{ Junio 2020 } & Mujer & 0 & 4,000 & 4,688 & 7,131 & 6,875 & 87,240 \\
& Hombre & 0 & 4,470 & 4,861 & 7,120 & 6,771 & 153,268 \\
\hline
\end{tabular}

Fuente: elaboración propia con los microdatos de la GEIH, DANE (2020).

Nota: durante los primeros tres meses de la pandemia, marzo, abril, y mayo; la GEIH sufrió una serie de modificaciones en cuanto al volumen y tipos de preguntas, entre las cuales no se encontraba los ingresos de asalariados e independientes.

Para las mujeres independientes el escenario parece ser más coherente de acuerdo al análisis teórico. Según la Tabla 2, para los meses analizados, los ingresos de las mujeres distaban mucho de su contraparte. Un ejemplo de ello se observa en el primer cuartil de la distribución, donde las mujeres ganan solo el 70 \% de los ingresos de los de su contraparte para el mes de enero, y $65 \%$ para el mes de febrero y junio.

En cuanto al ingreso promedio se puede observar un decrecimiento de los salarios femeninos frente al masculino tanto en febrero como en junio. Para el mes de enero del año 2020, la población femenina ganaba en promedio el $95 \%$ del ingreso de la población masculina, cifra que disminuyó a 89 \% para el mes de 
febrero. Luego de la pandemia, para el mes de Junio, las mujeres ganaban sólo el $82 \%$ del ingreso promedio por hora desu contraparte.

Un hecho que se destaca son los ingresos máximos de la muestra, en la cual vuelve a observarse una tendencia poco clara a través de los tres meses analizados. Como se mencionó anteriormente, estos resultados se encuentran influenciados por los datos atípicos de la muestra, sin embargo, es posible observar que, para el mes de enero, las mujeres ganaban en promedio 14 \% más que los hombres por hora. Esta cifra cambia, siendo en junio, tiempos de la COVID-19, los hombres quienes obtienen ingresos por horas superiores que las mujeres.

Tabla 2. Ingresos por horas de los independientes antes y durante la COVID-19

\begin{tabular}{cccccccc}
\hline Mes & Género & Min & 1 st $\mathrm{Q} u$. & Median & Mean & 3rd $\mathrm{Q} u$. & Max. \\
\hline \multirow{2}{*}{ Enero 2020 } & Mujer & 0 & 1,562 & 3,125 & 5,198 & 5,357 & 437,500 \\
& Hombre & 0 & 2,250 & 3,646 & 5,447 & 5,357 & 375,000 \\
\hline \multirow{2}{*}{ Febrero 2020 } & Mujer & 0 & 1,429 & 2,857 & 4,592 & 5,000 & 200,000 \\
& Hombre & 0 & 2,188 & 3,571 & 5,138 & 5,208 & 212,500 \\
\hline \multirow{2}{*}{ Junio 2020 } & Mujer & 0 & 1,500 & 2,778 & 4,247 & 4,861 & 166,667 \\
& Hombre & 0 & 2,188 & 3,500 & 5,165 & 5,000 & 234,375 \\
\hline
\end{tabular}

Fuente: elaboración propia con los microdatos de la GEIH, DANE (2020).

Nota: durante los primeros tres meses de la pandemia, marzo, abril, y mayo; la GEIH sufrió una serie de modificaciones en cuanto al volumen y tipos de preguntas, entre las cuales no se encontraba los ingresos de asalariados e independientes.

Respecto al salario promedio por tipo de actividad en tiempos de la COVID-19, se pueden observar varios elementos a destacar. El primero de ellos es que las actividades donde se muestra más equidad salarial por género son los empleados de gobierno y de empresa particular; incluso en estos dos casos, la población femenina goza de una remuneración promedio ligeramente mayor a los de su contraparte. En los trabajadores por cuenta propia se puede percibir una brecha de salario de $18,5 \%$ a favor de los hombres. En cuanto al trabajo doméstico, una actividad que ha sido feminizada historicamente, las brechas ascienden a 26,6 \%, siendo la mujer con menor salario. Lo anterior es bastante paradójico, teniendo en cuenta que para el mismo mes el 96 \% de las personas que trabajan en esta actividad son mujeres, según puede observarse en la Figura . 
Por otro lado, la población masculina que labora como jornalero o peón ganan 229.272 pesos colombianos más que la población femenina, llegando a una brecha de $38,1 \%$. Finalmente, las mujeres que son empleadoras ganan en promedio el 48,9 $\%$ de los ingresos de los hombres en esta misma actividad (ver Figura ).

Figura 4. Salario promedio por tipo de actividad en junio 2020

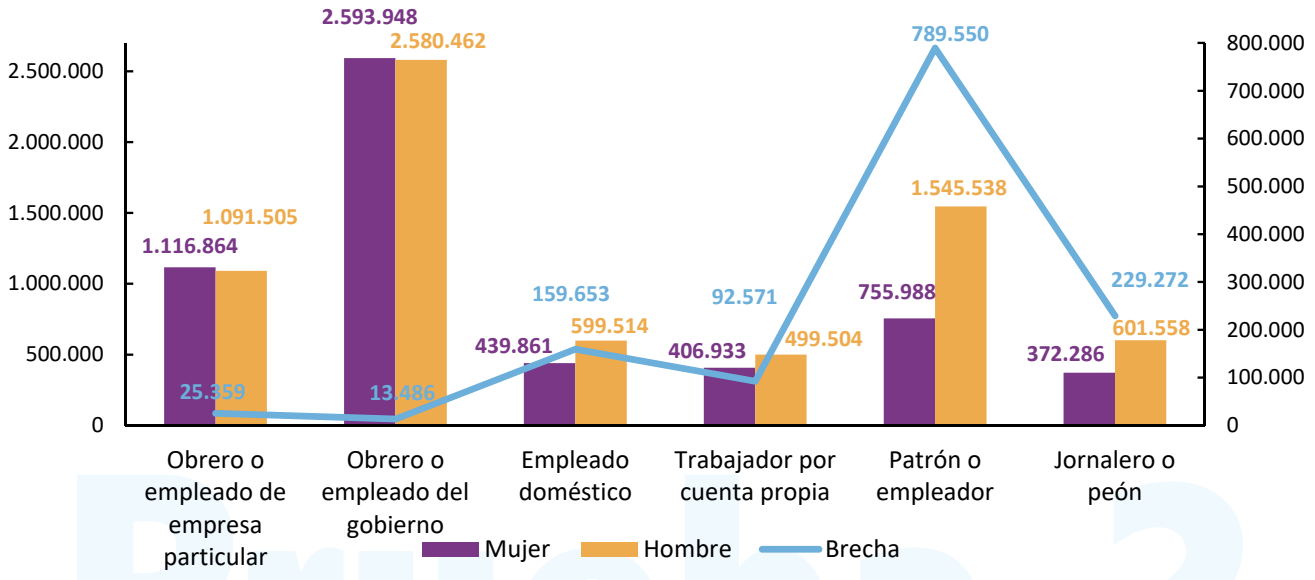

Fuente: elaboración propia con los microdatos de la GEIH. DANE (2020).

Nota: se eliminaron los tipos de actividad que no tienen una remuneración tales como: trabajador familiar sin remuneración y trabajador sin remuneración en empresas o negocios de otros hogares.

\subsection{Género e inactividad}

Al examinar los datos correspondientes a la inactividad, se evidencia una tendencia creciente en la cantidad de población inactiva, esta tendencia es compartida tanto en hombres como en mujeres, siendo estas últimas las que presentan mayores cifras de inactividad, según puede observarse en la Ilustración 5. Se puede ver como en los dos años, en los distintos trimestres analizados, la inactividad aumentó en ambos géneros. 
Figura 5. Inactivos 2019-2020 (miles)

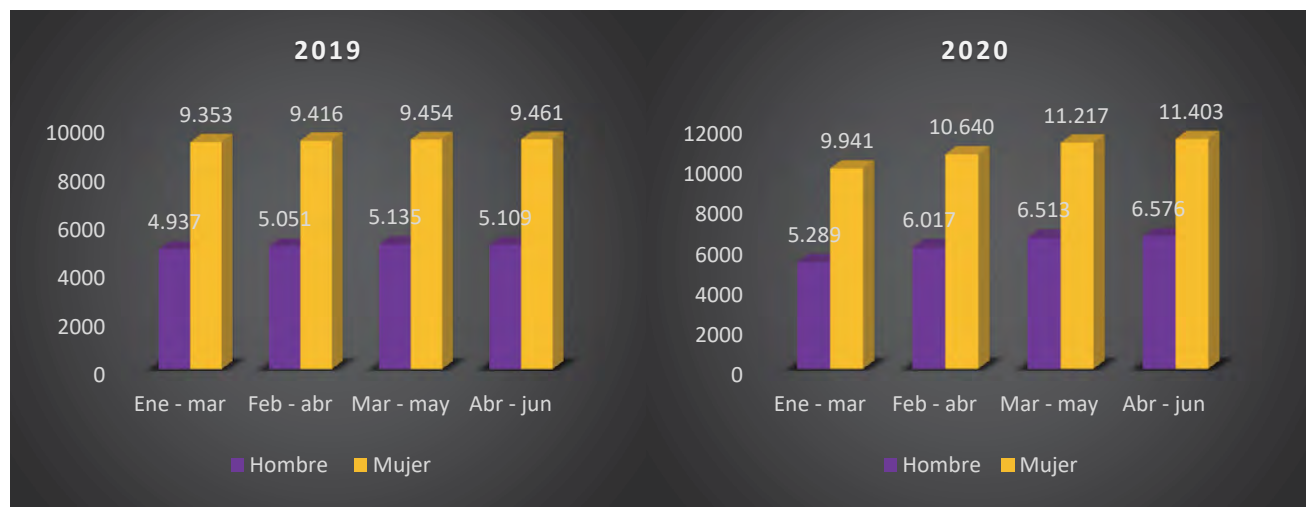

Fuente: elaboración propia con datos de la GEIH. DANE (2020).

Si se revisa la tasa de inactividad ${ }^{5}$ por género para los dos años y trimestres, se puede apreciar como la diferencia en estas es bastante amplia (ver ilustración 6). Par el año 2019, la tasa de inactividad promedio de las mujeres fue 11,1 pps más alta que la de los hombres, mientras que para el año 2020, esta diferencia aumentó a 11,8 pps, lo que evidencia en choque macroeconómico causado por la COVID-19. La diferencia más alta entre periodos se observa en el trimestre abril-junio, cuando en 2019 la diferencia entre tasas de hombres y mujeres fue de 11,1 pps, mientras que en 2020 esta diferencia fue de 12,1 pps.

Figura 6. Tasa de inactividad 2019-2020
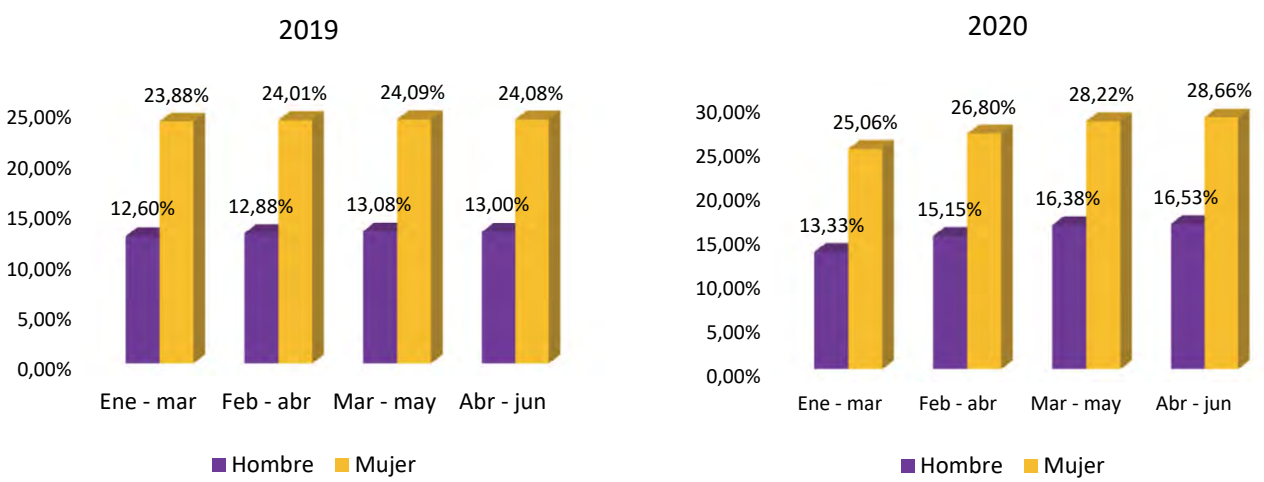

Fuente: elaboración propia con datos de la GEIH, DANE (2020)

\footnotetext{
5 La tasa de inactividad se calcula como el porcentaje de la Población Económicamente Inactiva sobre la Población en Edad de Trabajar (PEI/PET).
} 
Las razones para el mayor incremento en la inactividad de las mujeres en comparación con los hombres están asociadas con el aumento en el cuidado doméstico y el trabajo no remunerado producto del confinamiento, cierre de las escuelas y guarderías, que ha recargado las responsabilidades del hogar en la mujer. De hecho, al revisar la proporción de mujeres inactivas que se dedican a los oficios del hogar, se obtiene un promedio de $62,3 \%$ para 2020, mientras que los hombres solo participan con un promedio de 13,9\%, como puede observarse en la Figura 6. Si se comparan los periodos de análisis con los de 2019, es evidente la disparidad de género y el aumento para el año 2020.

Figura 7. Inactivos en oficios del hogar (participación)

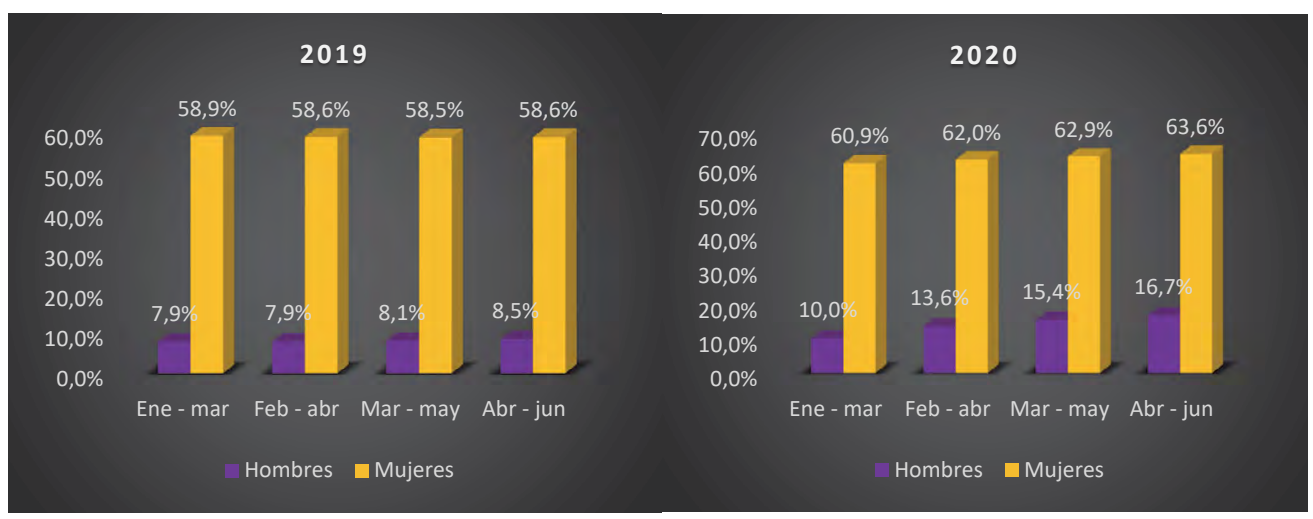

Fuente: elaboración propia con datos de la GEIH. DANE (2020).

Otro elemento a destacar de la población inactiva es el motivo principal por el cual dejó el último trabajo. Como se observa en la Figura 7 e Figura 8 la razón por la cual las mujeres dejaron su último empleo fue las "responsabilidades familiares". Tanto antes y después de la pandemia, el $98 \%$ de las personas que dejaron su trabajo a causa de las "responsabilidades familiares" fueron mujeres, un hallazgo que confirma la sobrecarga de trabajo doméstico y del cuidado dentro del hogar para la población femenina. Adicional, antes de la pandemia de salud, el 74 \% de las personas que renunciaban a su trabajo debido a que tenía condiciones insatisfactorias eran mujeres, cifra que disminuyó para junio del 2020 a 70 \%. Lo anterior podría estar sustentado en que las condiciones laborales para las mujeres suelen ser menos favorables y más precarias que para los hombres. 
Figura 8. Motivos principales por los cuales se renunció al trabajo durante la COVID-19 (junio-2020)

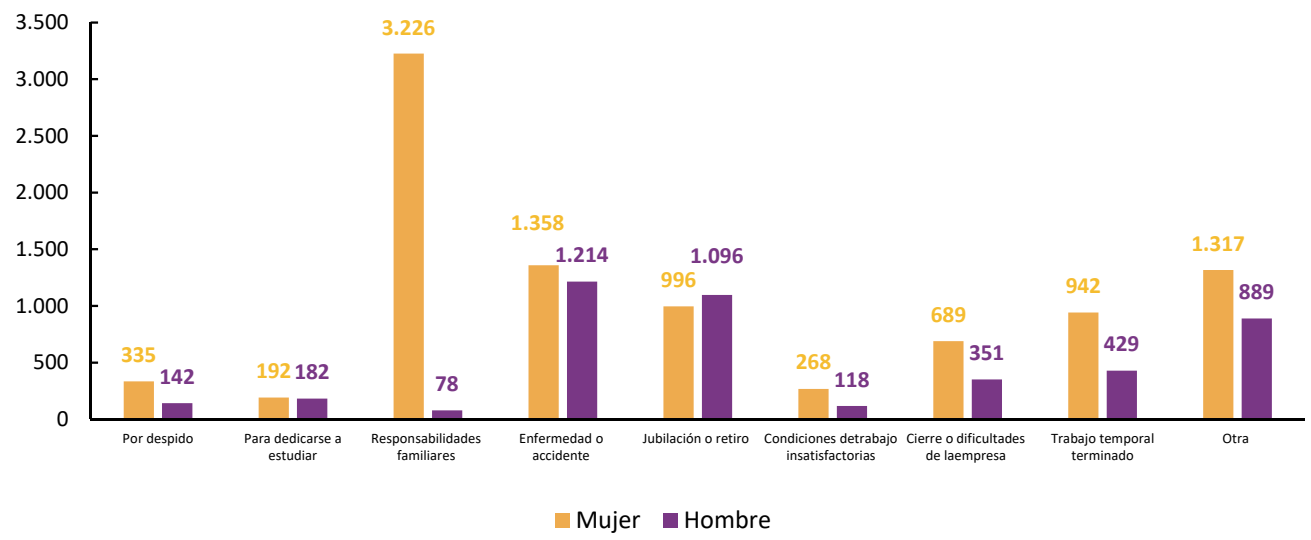

Fuente: elaboración propia con los microdatos de la GEIH. DANE (2020).

Figura 9. Motivos principales por los cuales se renunció al trabajo antes de la COVID-19 (enero 2020)

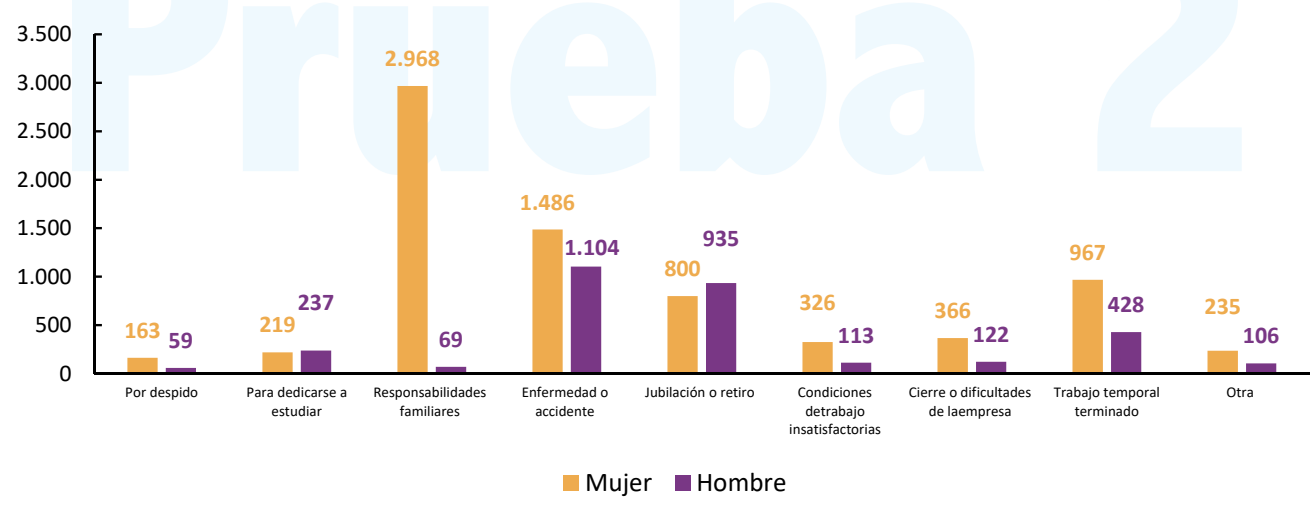

Fuente: elaboración propia con los microdatos de la GEIH. DANE (2020).

\subsection{Género, sectores productivos y segregación horizontal}

Las cifras relacionadas con los sectores económicos y la participación por género muestran en general una reducción de la población ocupada en los distintos sectores, esto, ocasionado por la contracción en la producción nacional que se traduce en la reducción del empleo. Pese a que el gobierno nacional ha implementado diversas estrategias de corte financiero para el sostenimiento de las empresas y los empleos, el panorama agregado muestra que la pérdida de empleos ha sido inevitable debido 
al choque macroeconómico, con mayor incidencia en algunos sectores considerados vulnerables, dada la naturaleza de la crisis sanitaria que afecta a los sectores productivos con mayor exposición al contacto físico.

El análisis por sector económico desagregado por género revela que para hombres y mujeres la participación en los distintos sectores productivos se ha visto reducida, no obstante, es importante resaltar que existen sectores que han sido afectados más que otros y que acogen a una alta proporción de mano de obra femenina. De acuerdo con Herrera-Idárraga y Tribín (2020), se han identificado al menos 31 sectores vulnerables, debido en gran parte a la imposibilidad de teletrabajo o porque son poco necesarios para operar en las crisis de salud, estos sectores cuentan con una mayor proporción de mano de obra femenina. Dentro de estos, los más importantes son: Comercio al por menor, Hoteles y restaurantes, Servicios domésticos, Otros servicios, Otras actividades empresariales, Prendas de vestir y Actividades inmobiliarias y de alquiler ${ }^{6}$. De acuerdo con la información para el trimestre móvil abril-junio, que se muestra en la Figura 9, algunos de estos sectores, aunque corresponden a revisiones CIIU distintas ${ }^{7}$, muestran relación con los sectores identificados por Herrera-Idárraga y Tribín (2020), como más vulnerables. Se observa como los sectores con mayor cantidad de ocupación femenina son: actividades artísticas, entretenimiento, recreación y otras actividades de servicios; administración pública y defensa, educación y atención de la salud humana; alojamiento y servicios de comida; actividades profesionales, científicas, técnicas y servicios administrativos; y actividades financieras y de seguros. En la Figura 10 por su parte se muestra la participación de cada una de las ramas o actividades económicas solo para la población femenina ocupada; entre estas se destacan el comercio y reparación de vehículos con 22,1 \% del total femenino; administración pública y defensa, educación y atención de la salud humana con 17,9\%; actividades artísticas, entretenimiento, recreación y otras actividades de servicios con 12,4\%; y alojamiento y servicios de comida con $11,2 \%$.

En línea con lo anterior, resulta importante verificar si estos sectores vulnerables identificados por Herrera-Idárraga y Tribín (2020) como vulnerables y que albergan mayoritariamente población femenina, han sido realmente afectados por la crisis derivada de la COVID-19, y en consecuencia han tenido un impacto diferenciado en el mercado de trabajo por género. Para esto, se procede a revisar los datos relacionados con la producción agregada en sus diferentes ramas de actividad económica, que corresponden a 12 Secciones de la CIIU revisión 4 A.C.

Los sectores corresponden a la desagregación a cuatro dígitos de la CIIU revisión 3. A.C.

7 Las cifras se presentan con revisiones CIIU distintas dada la disponibilidad de las mismas. 
De acuerdo con la Figura 11 la variación trimestral del PIB para las diferentes ramas de actividad económica ha sido para la mayoría de estas, negativa. Las ramas que han presentado las mayores variaciones son actividades artísticas, de entretenimiento y recreación y otras actividades de servicios; actividades de los hogares individuales en calidad de empleadores; actividades no diferenciadas de los hogares individuales como productores de bienes y servicios para uso propio con $-34,86 \%$ con respecto al primer trimestre de 2020; seguido de comercio al por mayor y al por menor; reparación de vehículos automotores y motocicletas; transporte y almacenamiento; Alojamiento y servicios de comida con -33,75\%; Industrias manufactureras con $-21,60 \%$ y actividades profesionales, científicas y técnicas, actividades de servicios administrativos y de apoyo con $-11,80 \%$.

Figura 10.Rama de actividad económica y distribución por sexo trimestre móvil abril-junio 2020 (miles)

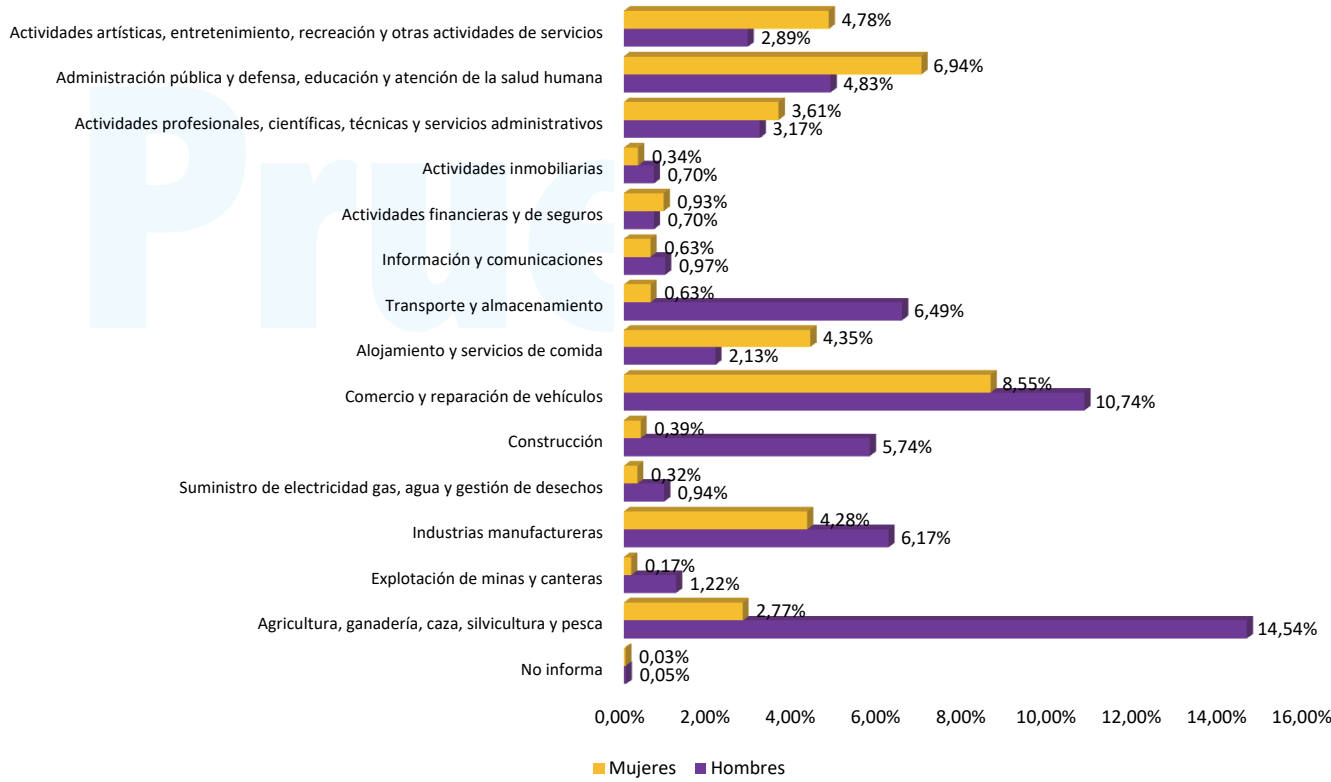

Fuente: elaboración propia con datos de la GEIH. DANE (2020).

Tan solo dos sectores obtuvieron variaciones positivas: actividades financieras y de seguros y actividades inmobiliarias. Como se puede apreciar en este gráfico. los sectores que poseen mayor cantidad de mano de obra femenina han sido los más afectados por la COVID-19, en consecuencia, la producción se ha reducido, lo que 
ha provocado el cierre de empresas y la pérdida de empleos para un gran número de mujeres en el mercado laboral.

Figura 11. Participación de la fuerza de trabajo femenina por rama de actividad económica. CIIU 4 Rev. A.C

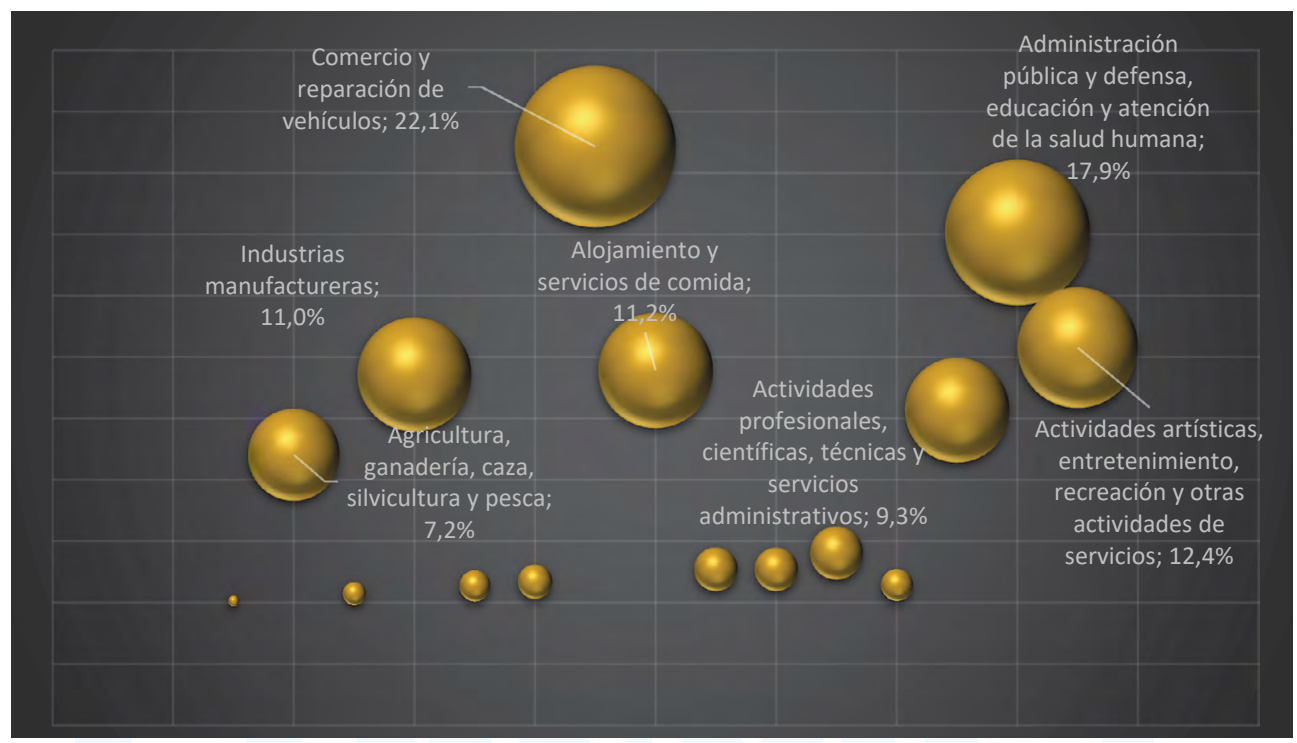

Fuente: elaboración propia con datos de la GEIH. DANE (2020). 


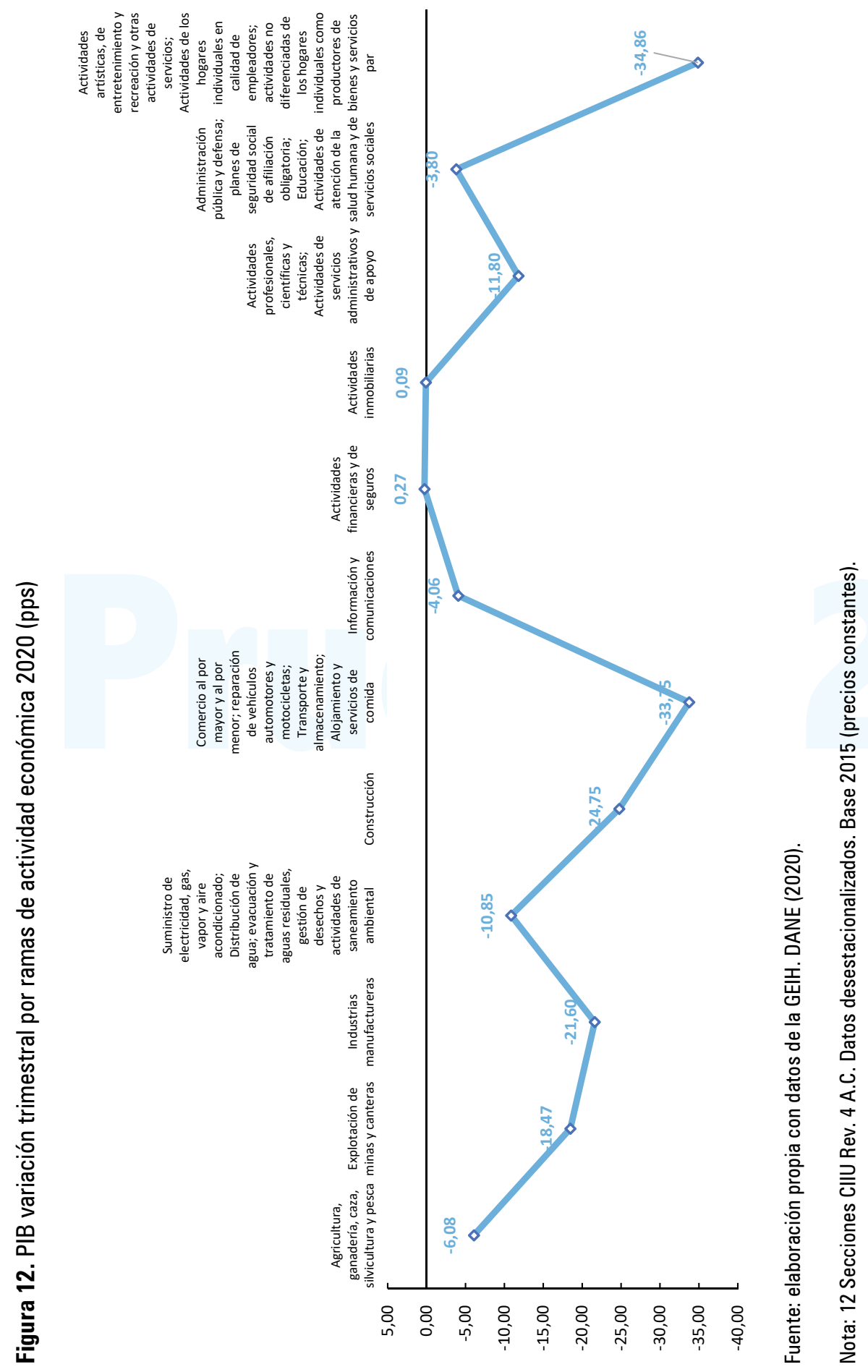




\section{CONCLUSIONES}

Este trabajo realizó un análisis de la situación actual y el rol de la mujer en el mercado laboral colombiano, en el escenario derivado de la COVID-19. La crisis de salud mundial ha ocasionado efectos nocivos en los mercados de trabajo de los distintos países, incrementando de cierta forma las brechas existentes en las naciones con deficiencias e imperfecciones en los mercados laborales, imperfecciones por las cuales el mercado de trabajo colombiano se caracteriza.

Una gran variedad de estudios han analizado las características y los indicadores de empleo femenino, encontrando que, tradicionalmente, este grupo poblacional, presenta cifras de empleo más bajas en comparación con los hombres, trabajos precarios e inserción en sectores económicos de baja productividad; dada la naturaleza de la actual crisis, donde se han visto afectados sectores intensivos en mano de obra femenina como los servicios, es posible señalar que los efectos de la COVID-19 han contribuido a incrementar la brecha de género ya existente en el mercado de trabajo colombiano.

En cuanto a la brecha de desempleo, los hallazgos sugieren un incremento significativo a favor de los hombres, ubicándose en el trimestre abril-junio de 2020 en $-7,25 \%$. La tasa de desempleo femenina para este mismo trimestre fue de 24,62 $\%$, mientras que el indicador para los hombres fue de $17,37 \%$. Se observa como de forma general, para todos los trimestres entre 2019 y 2020 la brecha ha aumentado.

El análisis para la ocupación indica un descenso agregado tanto en hombres como en mujeres, la brecha de ocupación se ha mantenido en niveles similares a los registrados en 2019 (22 \%), no obstante, al tener una menor participación en el mercado laboral, se espera un mayor impacto nocivo en la población femenina en comparación con la masculina. Al examinar los ingresos por hora de forma agregada diferenciando por sexo, no se encontraron grandes diferencias para el caso de los asalariados; sin embargo, el análisis de acuerdo con la posición ocupacional muestra que, para las mujeres en condición de independientes, entre enero y febrero de 2020 el salario de las mujeres independientes correspondía al 95 \% y $89 \%$ del salario de los hombres, respectivamente. Durante la pandemia, los ingresos por hora para las mujeres disminuyeron respecto a su contraparte, llegando a ser solo el $82 \%$ de los ingresos de los hombres.

En lo asociado a la inactividad, en el trimestre móvil abril-junio de 2020 se amplió la brecha de inactividad, más mujeres pasaron a ser inactivas dedicándose en gran proporción a los oficios del hogar: $64 \%$ de las mujeres inactivas se dedican a las tareas domésticas, mientras que solo un $17 \%$ de los hombres contribuye a estas 
labores. Estos resultados evidencian la fuerte desigualdad persistente en los roles de género, y, en consecuencia, es una de las mayores barreras para la inserción y permanencia de las mujeres en el mercado laboral.

Finalmente, en cuanto la distribución por ramas de actividad económica, se pudo constatar que los sectores que han presentado variaciones negativas en la producción agregada como las Actividades artísticas, de entretenimiento y recreación y otras actividades de servicios; Actividades de los hogares individuales en calidad de empleadores, Actividades no diferenciadas de los hogares individuales como productores de bienes y servicios para uso propio; Comercio al por mayor y al por menor; reparación de vehículos automotores y motocicletas, Transporte y almacenamiento; Alojamiento y servicios de comida; Industrias manufactureras y Actividades profesionales, científicas y técnicas y Actividades de servicios administrativos y de apoyo, son las que albergan la mayor participación de mano de obra femenina, por lo que es más probable que las mujeres se vean más afectadas que los hombres en términos de pérdida del empleo. Ante la imposibilidad de teletrabajo o bien la necesidad del cuidado doméstico a causa del confinamiento, es de esperarse que se sigan ampliando las brechas de género en el mercado laboral femenino.

Los resultados aquí expuestos pueden servir como insumo para el diseño de políticas públicas con enfoque de género, orientadas a abordar esta problemática que ha persistido por años y que se ha agudizado con la emergencia sanitaria actual. Debido a las limitaciones relacionadas con los datos, en este trabajo no fue posible realizar análisis de tipo econométrico; no obstante, otros trabajos en la misma línea podrían dirigirse a realizar modelaciones econométricas y análisis predictivos que contribuyan a conocer de manera exacta cuales han sido los efectos puntuales de la crisis sanitaria en el mercado laboral femenino, en la medida que los datos estén disponibles.

\section{BIBLIOGRAFÍA}

Adams-Prassl, A., Boneva, T., Golin, M. y Rauh, C. (2020). Inequality in the Impact of the Coronavirus Shock: Evidence from Real Time Surveys. IZA Institute of labor Economics. Discussion Paper. 13183.

Alon, T., Doepke, M., Olmstead-Rumsey, J. y Tertilt, M. (2020). The Impact of COVID-19 on gender equality. NBER Working Paper 26947. 10.3386/w26947

Avendano-Gelves, L. F. (2011). Segregación laboral y discriminación salarial de género en Colombia: el caso de las trece áreas metropolitanas, 2001, 2005 y 2009. Revista de economía del Caribe, 8, 202-231. 
Bello-Bello, M. S. y Sepúlveda-Carrillo, P. I. (2016). Segregación laboral por sexo en las grandes ramas de la actividad económica en Colombia 2008-2013. [Trabajo de maestría. Universidad de La Salle]. Repositorio Ciencia Unisalle. https://ciencia.lasalle.edu.co/maest_gestion_desarrollo/99

Cerquera-Losada, O. Arias-Barrera, C. J. y Murcia-Arias. J. P. (2019). Diferencial salarial por género: un análisis comparativo entre departamentos de la costa atlántica colombiana. Revista virtual Universidad Católica del Norte, 56, 109-125.

Cerquera-Losada, O. Arias-Barrera, C. J. y Prada-Hernández, J. F. (2020). La brecha salarial por género en Colombia y en el departamento de Caldas. Ánfora, 27 (48),113-136. https://doi. org/10.30854/anf.v27.n48.2020.671

Chacón-Bejarano. F. D. y Vanegas-Triana. S. C. (2019). Efecto techo de cristal en Colombia: causas. consecuencias y algunas soluciones propuestas. [trabajo de grado. Universidad de La Salle]. Repositorio Ciencia Unisalle. https://ciencia.lasalle.edu.co/economia/915

Departamento Administrativo Nacional de Estadística y Organización de Naciones Unidas Mujeres (2019, octubre). Boletín estadístico de empoderamiento económico de las mujeres en Colombia. https:/www.dane.gov.co/files/investigaciones/genero/publicaciones/Boletin-Estadistico-ONU-Mujeres-DANE.pdf

Departamento Administrativo Nacional de Estadística (2019). Principales resultados del mercado laboral. Total nacional y 13 ciudades y áreas metropolitanas. https://www.dane.gov.co/files/investigaciones/ boletines/ech/ech/pres_web_empleo_resultados_dic_19.pdf

Farré, L., Fawaz, Y., González, L. y Graves. J. (2020). How the COVID-19 Lockdown Affected Gender Inequality in Paid and Unpaid Work in Spain. Iza Institute of Labor Economics Discussion Paper, 13434.

Fleta-Asín, J. y Pan. F. (2017). Segregación horizontal y vertical de género en el profesorado. Acciones e Investigaciones Sociales, 37, 187-214. https://doi.org/10.26754/ojs_ais/ais.2017372192

Flores, L. y Salas, I. A. (2015). Las brechas de género en la calidad del empleo en México: una valoración basada en modelos de lógica difusa. Análisis Económico, 30 (75), 89-112.

IMF- International Monetary Found (2020). World Economic Outlook. https://www.imf.org/es/Publications/WEO/Issues/2020/04/14/weo-april-2020

Galvis, L. A. (2010). Diferenciales salariales por género y región en Colombia: Una aproximación con regresión por cuantíles. Documentos de trabajo sobre Economía Regional y Urbana, 131, Banco de la República de Colombia.

Garzón-Lince, D. V. y Ulloa-Cantillo. J. (2016). Participación femenina en altos cargos directivos: perfil y estilo de liderazgo en empresas del sector privado en Colombia para la última década 2005-2015. [Trabajo de grado. Universidad de La Salle]. Repositorio Ciencia Unisalle. https://ciencia.lasalle.edu. $\mathrm{co} /$ cgi/viewcontent.cgi?article $=1001$ Econtext $=$ negocios_relaciones

Herrera-Idárraga, P. y Tribín, A. M. (2020). Informe sobre ocupación. ¿Podrá la crisis actual aumentar la brecha de género en ocupación? Grupo de enfoque diferencial e interseccional Dirección General del DANE. https://www.dane.gov.co/files/investigaciones/genero/informes/Informe-ocupacion-genero-y-covid.pdf 
Hupkau, C. y Petrongolo, B. (2020). Work. care and gender during the Covid-19 crisis. A CEP Covid-19 analysis Paper 2. Centre for Economic Performance. London School of Economics and Political Science.

Kristal, T. y Yaish, M. (2020). Does the coronavirus pandemic level the gender inequality curve? (It doesn't). Research in Social Stratification and Mobility. 68 (100520). https://doi.org/10.1016/J. RSSM.2020.100520.

Martín, M. (2007). La mujer en la industria publicitaria. La segregación vertical en la comunicación comercial: techo de cristal y suelo pegajoso. Revista de Estudios de Comunicación, 22, 429-452.

Mejía-Lleras, D. (2018). Comparación de metodologías de medición de discriminación salarial por género en el mercado laboral colombiano. [Trabajo de maestría. Universidad Nacional de Colombia]. Repositorio Institucional UN. https://repositorio.unal.edu.co/bitstream/handle/unal/69080/ DiegoMej\%C3\%ADaLleras.2018.pdf?sequence $=1$ EisAllowed $=y$

Mendoza, D. y Melo, J. (2019). Maternidad e inserción laboral femenina en trabajos de condiciones precarias: una comparación entre Brasil y Colombia. Semantic Scholar. https://www.semanticscholar. org/paper/Maternidad-e-Inserci\%C3\%B3n-laboral-femenina-en-trabajos-Cuello-Vieira/ ea9ff9587ce6c7614d88a9f0de12b2bec1c264ee

Moreno, E. N. (2017). La economía invisible: división sexual del trabajo doméstico y de cuidado no remunerado y el uso del tiempo de las mujeres en Bogotá. [Tesis de Maestría, Universidad Nacional de Colombia].

Meza-Martínez, C. A. (2018). Discriminación laboral por género: una mirada desde el efecto techo de cristal. Equidad y Desarrollo, 32. 11-31. https://doi.org/10.19052/ed.5243

Oreffice, S. y Quintana-Domeque, C. (2020). Gender Inequality in COVID-19 Times: Evidence from UK Prolific Participants. IZA Institute of Labor Economics. Discussion Paper.13463.

Organización para la Cooperación y el Desarrollo Económicos (2020, 10 de junio). COVID-19 crisis in the MENA region: impact on gender equality and policy responses. (Tackling coronavirus (COVID 19). Contributing to a global effort). Organisation for Economic Cooperation and Development. http://www.oecd.org/coronavirus/en/

Palacios-Riaño, M. C. (2019). Feminización de las ocupaciones y diferencias salariales por género para Colombia urbana 2008-2016. [Trabajo de grado. Universidad de La Salle]. Repositorio Ciencia Unisalle. https://ciencia.lasalle.edu.co/economia/887

Torns, D. y Recio, C. (2012). Las desigualdades de género en el mercado de trabajo: entre la continuidad y la transformación. Revista de Economía Crítica, 1 (14). 178-202.

UNDP- United Nations Development Program (2020). Briefing note: the economic impacts of COVID-19 and gender inequality. Recommendations for policymakers. http://americalatinagenera.org/newsite/images/cdr-documents/2020/04/PNUD_GENERO_COVID19_ENG_FINAL_1.pdf 